\title{
الاطلاع العقلي المباشر على الحقائق البَدَهية
}

\section{د.فيصل بشير محمد الخراز}

\section{جامعة مصراتة}

\section{مقدمة:}

لا شك أن الوصول إلى الحقائق والمعارف من أهم سمات البشر الذي وهبه الله العقل ليستخدمه في الكشف والبحث عن المعرفة والحقيقة، والتي يسعى للوصولإليها ببذل ذهني شاق،

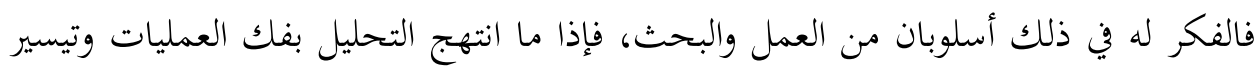
التعقيدات فهو العقل، وإن عمل على الإدراك المباشر أو الاطلاع المباشر على الحقائق البدهية فهو الحدس، فالعقل هو الذي يتبع أسلوب التحليل ويكابد للوصول إلى الحقيقة، ويعتمد على له الوصف والتحليل ويركن إلى التصورات العامة فإن الحدس فبعكس ذلك، فهو لا يحتاج إلى جُهـ ذهني، ويضعك تمامًا بكل يسر أمام الحقيقة وبعملية واحدة، فما يجده التحليل العقلي معقدًا

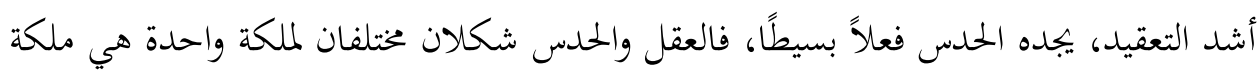
الفكر، هذه الملكة إذا ما ضاقت تسمى العقل، وإن اتسعت تسمى الححس، فما هذا الحدس؟

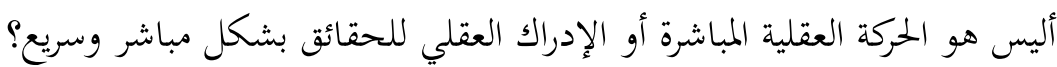

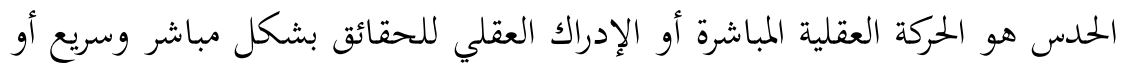
بمعنى آخر هو التخمين والتوهم أو التصور الذهني الذي يصدر عن نور العقل وحده. وتأتي إشكالية البحث في ان أصحاب المذهب الحدمسي ينطلقون من منطق التمرد على الاتحاه الحسي التجريبي الذي لا يؤمن في الوصول إلى المعرفة الحقة الا بما هو حسي فقط،

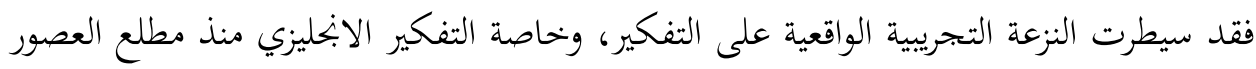
الحديثة، وبدأت في القرن السابع عشر عن توماس هوبزبواقعيته السياسية الأخلاقية، وعند فرنسيس بيكون بمنهجه التجريبي العلمي وعند جون لوك بنزعته التجريبية في فلسفته

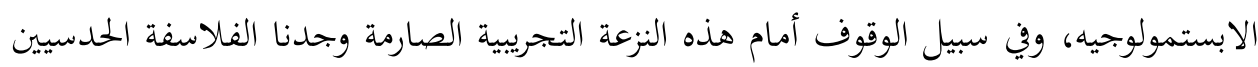


الذين يرون بسهولة الوصول إلى الحقيقة دونما الرجوع الم الوقائع أو حتى الاستدلال العقلي،

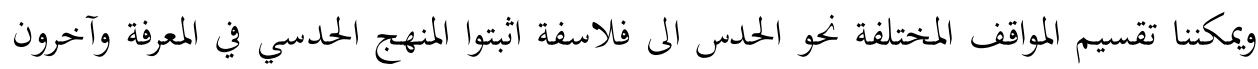
طبقوه في بحال الأخلاق وكذلك طبقه الفلاسفة في مبدإ الفن، والحدس الفني. وتأتي أهميته البحث في معرفة مفهوم الحدس والوصول إلى شرح تفصيلي للحدوس وأنواعها وتقسيم المواقف المختلفة نحو الحدس إلى فلاسفة اثتبوا المنهج الحمدسي في المعرفة وفلاسفة طبقوه في محال الأخلاق وآخرون طبقوه في محال الفن وهو ما يعرف بالحدس الفني. وقد قمت باتباع المنهج التحليلي فهو المخول له رصد حركة هذا العمل في صورة حركة جدلية مما يُمكِنِ القارئ من التحقق من هذا المفهوم من خلال تحليل أهم آراء هؤلاء الفلاسفة و تأثرهم بغيرهم من الفلاسفة ولا نكتفي بالمنهج التحليلي فحسب إنما نركن ايضًا إلى المنهج المقارن لما له من أهمية في إبراز الرؤى ووجهات النظر المختلفة لمؤلاء الفلاسفة وآخرين ممن تأثروا بهم.

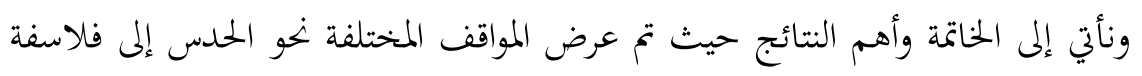

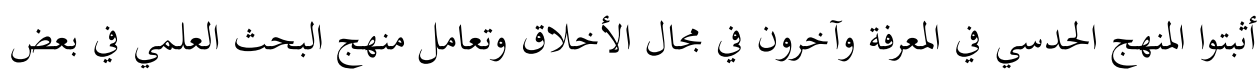
الحالات مع ما يسمى بالمعرفة المباشرة أو الحدسية.

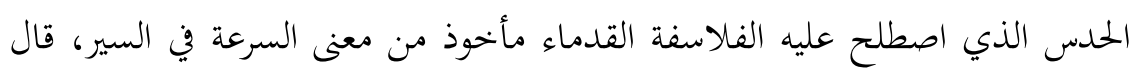

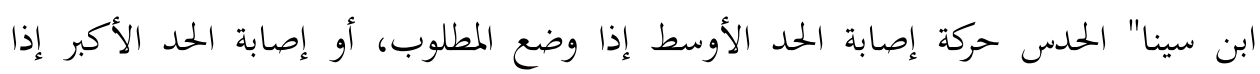
أصيب الأوسط، والجملة سرعة الانتقال من معلوم إلى بحهول (1). مفهوم الحمد:

إذا ما بحثنا في المعنى اللغوي فإننا بحد أن الحدس عرف في اللغة بأنه الظّن والتخمين، والتوهم في معاني الكلام والأمور والنظر الخنفي. وقال الجرجاني في تعريفاته، الحدس هو سرعة انتقال الذهن من المبادئ إلى المطالب

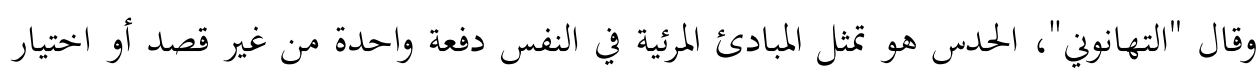

1- جميل صليبيا، المعجم الفلسفي، مادة "حدس" ص451. 
سواء بعد طلب أولا، فيحصل المطلوب (( والمقصود بالحركة وسرعة الانتقال تمثل المعنى في النفس دفعة واحدة في وقت واحد، كأنه وحي مفاجئ أو وميض برق )( (1). ويرى البعض الآخر أن الحدس فعل للذهن يستنبط به لذاته الحد الأوسط، والذكاء قوة

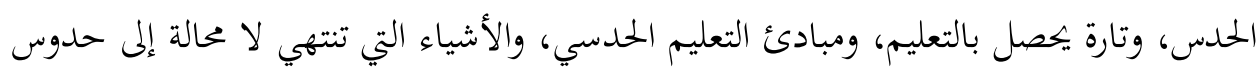
استنبطها أرباب تلك الحدوس ثم أدوها إلى المتعلمين (2). 1- 1 الحدس المعرفي عند ديكارت:

وجد من عبر عن هذا الحدس وقدم له شروحًا في فلسفته هو الفيلسوف

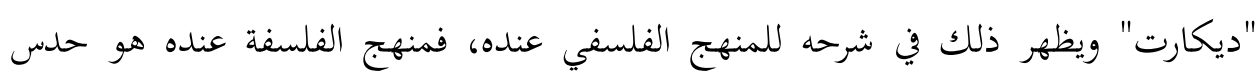
المبادئ البسيطة، واستنباط قضايا جديدة من المبادئ لكي تكون الفلسفة جملة واحدة (3). ولذلك يرفض ديكارت أن يكون الاستقراء هو مصدر اليقين، حيث أن الاستقراء عنده لا يصل إلا إلى معارف متفرقة إن جمعت بعضها إلى بعض ألفت علمًا مهلهالاً ملفقًا لا لاءعراء ندري من أين نلتمس له اليقين، ولكن علامة اليقين ووضوح المعاني وتسلسلها نصل إليها في الرياضيات التي تمضي من البسيط إلى الواضح المركب، وهذا المنهج هو المنهج الوحيد المشروع، لأن العقل واحد ويسير على نحو واحد في جميع الموضوعات ويؤلف علمًا واحدًا هو العلم الكلي، فلا تتمايز العلوم فيما بينها بموضوعاها ومناهجها، ولكنها وجهات مختلفة لعقل واحد يطبق منهجًا واحدًا ولا خير يرجى من القياس الأرسطي، والقياس الحقيقي عنده هو سلسلة من الحدوس تتقدم من حد إلى حد بحركة متصلة، فيربط العقل بين حدود لم تكن علاقتها واضحة أول الأمر، حتى إذا ما انتهى في الاستنباط إلى غايته، رد البمهول إلى المعلوم، أو المركب إلى البسيط أو الغامض إلى الواضح، والحركة العقلية هنا ثانوية، فإن مبدأها حدس ومنتهاها

$$
\text { 1 - جميل صليبيا، المعجم الفلسفي، مادة "حدس" ص451. }
$$

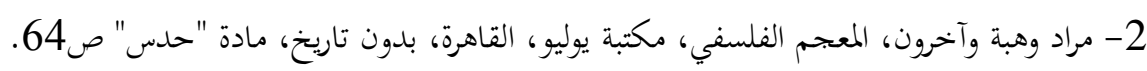

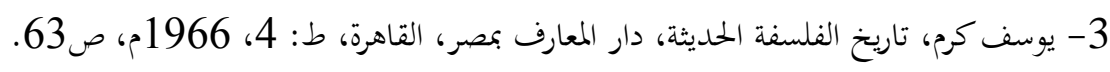


والحدس عند ديكارت هو الإطلاع العقلي المباشر على الحقائق البدهية ولذلك نجده

يقول، (( أنا لا أقصد بالحدس شهادة الحواس المتغيرة ولا الحكم الخداع لخيال فاسد المباني، إنما أقصد به التصور الذي يقوم في ذهن خالص منتبه، بدرجة من السهولة والتمييز لا يبقى معها بحال للريب، أي التصور الذهني الذي يصدر عن نور العقل وحده، ومعنى ذلك أن الحدس عنده عمل

عقلي يدرك به الذهن حقيقة من الحقائق يفهمها بتمامها في زمان واحد، لا على التعاقب )(2). وقد وضع ديكارت لنفسه منهجًا في سبيل الوصول إلى اليقين وأعلى من شأن الرياضة في تأسيس هذا المنهج معبرًا أن الرياضة تبدأ بما يسميه البداهة التي تبدأ في الذهن وهي أساس الفكر والنظر، فنجده يقول، (( أنا لا أتلقى أي شيء على أنه حق، ما لم أتبين بالبداهة أنه كذلك )) (3). ولقد حاول ديكارت أن يطبق المنهج الرياضي على قضايا العلم فذهب إلى تأكيد اليقين الرياضي فأصبح هو النموذج الأمثل للمعارف اليقينية الجلية المتميزة، ورأى أنه لن تكتسب المعارف صفة الصدق الرياضي ولن يعدل يقينها يقين العلوم الرياضية ما لم تتوفر فيها خصائص وشروط الفكر الرياضي، وهي، أ. وجود المعاني المتسمة بالوضوح والتمييز ومعنى هذا أن تكون على مستوى بداهة

مطلقة. ب. التوجه المستمر من المعاني إلى الأشياء وهذا يعني ألا نلصق بالأشياء صفات لا علاقة بالبداهة بها، فلا ننسب للأشياء إلا ما نستطيع إدراكه إدراكًا بدهيَّا.

$$
\text { 2- 1- يوسف كرم، مرجع سابق ص63. }
$$

3- راوية عبد المنعم عباس، ديكارت والفلسفة العقلية، دار المعرفة الجحامعية، الإسكندرية، 1990م، ص89. 


\section{ج. تنظيم وترتيب أفكارنا في نسق خاص (1).}

ويؤكد ديكارت على دور الحدس في العملية المعرفة التي توصلنا إلى كشف الحقائق واليقين المعرفي فيقول، (( إن كل العمليات العقلية التي تمكننا من الوصول إلى معرفة الأشياء دون أن نخشى الوقوع في الخطأ لا تتعدى عمليتين هما الحدس والاستباط )) (2). وعرف ديكارت الحدس في هذا السياق بقوله إنه (( الرؤية العقلية المباشرة التي يدرك ها العقل بعض الحقائق التي لا نستطيع نكراها وهو لا يقتصر على المفاهيم والأفكار، بل يحتوي على كل المعاني والمبادئ التي لا تقبل الشك، والاستباط هو عملية عقلية تمكننا من استخلاص فكرة من أفكار أخرى نعرفها على وجه اليقين، والاختلاف بين الحدس والاستنباط يكمن في أن الحدس يدرك الطبائع البسيطة والحقائق الثابتة، والاستباط عملية عقلية تنطوي على حركة أو تعاقب، وأن المبادئ الأولى تعرف بالحدس وحده، والنتائج البعيدة بواسطة الاستنباط (3)

ومن ثم فإن المنهج الديكارتي يقوم على فعلين هما الحدس والاستنباط وهما يرجعان إلى فعل واحد، فالاستنتاج ليس إلا سلسلة من الحدوس لما بين حقيقة وأخرى من ارتباط منطقي وضروري (4).

$$
\text { والأمور التي يدركها العقل بالحدس عند ديكارت ثلاثة أنواع، }
$$

ب. الحقائق الأولية التي لا تقبل الشك، كعلمي أني موجود، لأني أفكر.

$$
\text { 1- 1 راوية عبد المنعم عباس، مرجع سابق ص87. }
$$

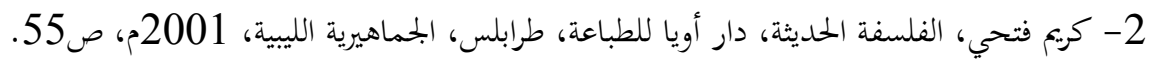

$$
\text { 3- 3- نفسه ص- نفسه. }
$$


ج. المبادئ العقلية التي تربط الحقائق بعضها ببعض، كعلمي أن الشيئين المساويين

لشيء ثالث متساويان لذلك سمى ديكارت هذا الحلدس نورًا طبيعيًا أو غريزة عقلية(1).

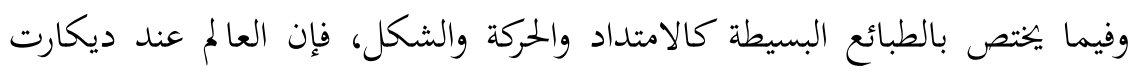

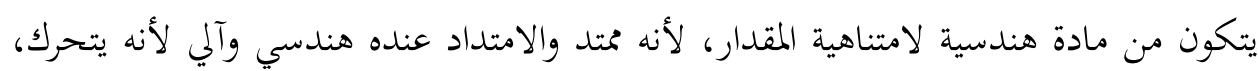
وكمية الحركة ثابتة فيه فيمتد الامتداد إلى كل ما هو موجود في الطبيعة، وهو بذلك يكون متحدًا بالمادة وأن أساس العالم ووجودهد في الحركة والامتداد (2).

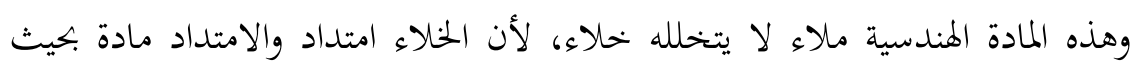
تفسر كل حركة بأن الجسم المتحرك يطرد الجسم المجاور له ليحل في مكانه، فالحركة في العالم

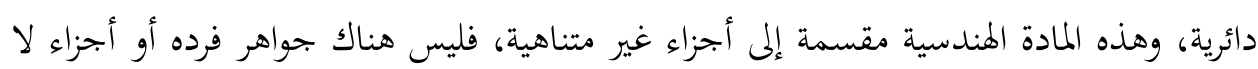

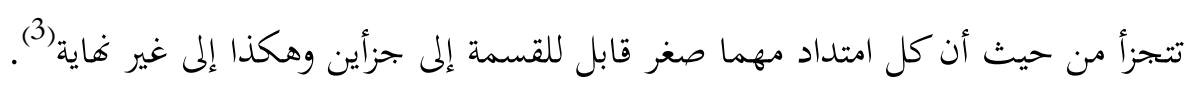

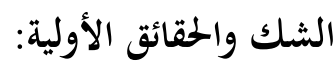

ومن أجل الوصول إلى الحقائق الأولية التي لا تقبل الشك والتي تعتمد على الحدس الحس

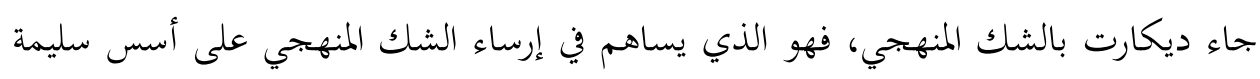
حيث قال، (( إننا لكي نبحث عن الحقيقة ينبغي أن نشكك في كل ما يصادفنا من أشياء ولو

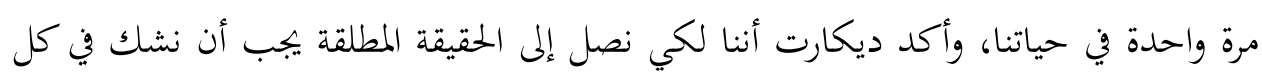
شيء موجود وأن نترك كل المعتقدات، وكل ما ورثناه من أفكار عن طريق الفكر الشائع أو أي سلطة، حتى نصل إلى الذوق العقلي، حيث يقول "ديكارت" العقل أعدل الأشياء قسمة بين (الناس () (4)

1- جميل صليا، مرجع سابق ص452، 1453.

2- علي عبد المعطي، دراسات في الفلسفة العامة، دار المعارف الجامعية، الإسكندرية، 153 مرئ 1991م، ص427.

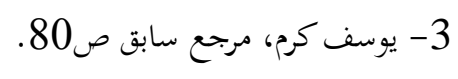

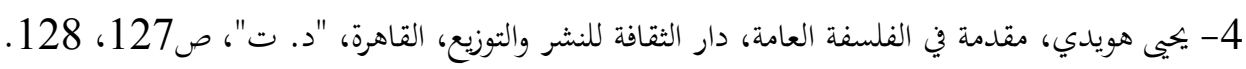


ولقد بالغ ديكارت في شكه للوصول إلى الحقيقة اليقينية فنجده بداية شك في كل

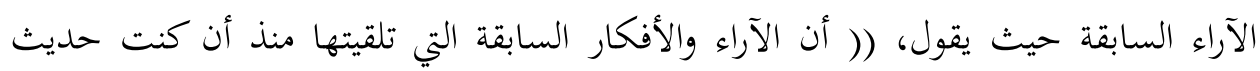

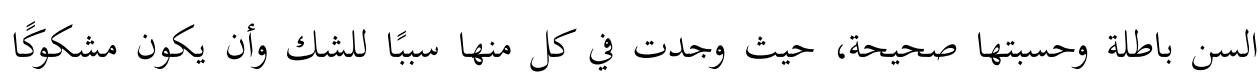
فيها () (1)

ثم سرعان ما انتقل في شكه إلى الحواس فيقول، (( أنا أشك في الحواس لأها خدعتني،

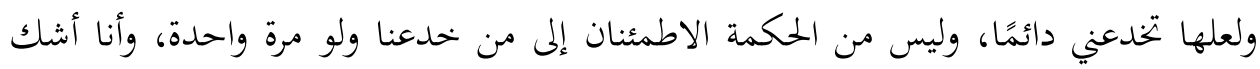

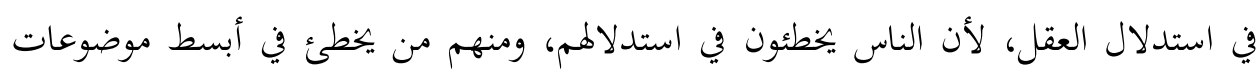

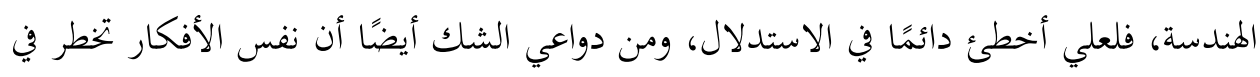

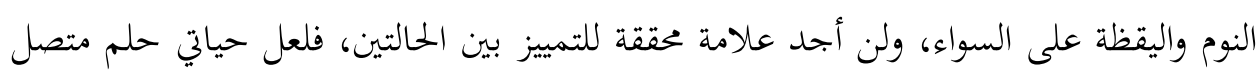
أي لعل اليقظة حلم منسق () (2).

وينتهي ديكارت إلى الحقيقة اليقينية التي يدركها بحدس عقلي مباشر وهي إدراك وجوده

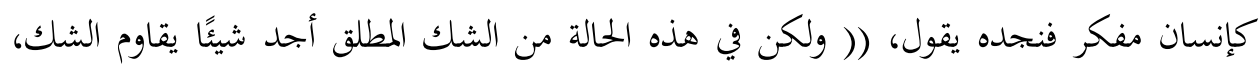

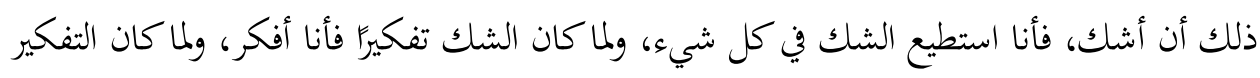

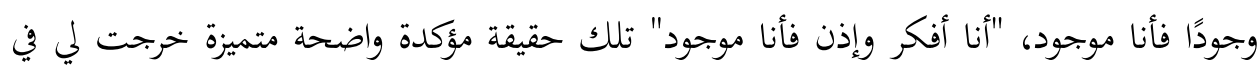

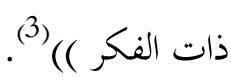

والتفكير هنا عند ديكارت لا يعني الاستدلال من شيء معلوم إلى شيء بمهول، وإنما معناه كل العمليات العقلية التي تعرضت للإنسان، وإن "أنا أفكر" تعني أني أحس وأشك داءك وأدرك 1- ديكارت، التأملات في الفلسفة الأولى، ترجمة عثمان أمين، مكتبة الأنخلو المصرية، القاهرة، "د. ت"،

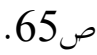

$$
\begin{aligned}
& \text { 2- يوسف كرم، مرجع سابق ص67.66. } \\
& \text { 3- نفسه ص67. نوس كرم. }
\end{aligned}
$$


وأتصور وأتذكر وأثبت وأنكر وأرغب ولا أرغب وأعتقد، وأنا موجود تعني أن الموجود أمر ثابت ولكنه مرتبط بالتفكير وإذا انقطعت عن التفكير، فإنني انقطعت عن الوجود من خلال هذا يسلم بأنه شيء يفكر أي أنه روح وإدراك وعقل (1). وهكذا فإن الكوجيتو الديكارتي عبارة عن قضية ذاتية مخضة يقرر صدقها ويقينها الحدسي(2)، أي لا تحتاج في نظر ديكارت إلى الحد الأوسط في القياس، ولكن هناك من يرفض هذه المعرفة المباشرة وهذا ما بنحه عند هيجل. 2.

يعترض هيجل على هذه المعرفة الحدسية والتي يعتبرها أصحابها أها مباشرة دون توسط. فيقول هيجل، أن المبدأ الذي أعلن عنه "ديكارت" أنا أفكر، إذن أنا موجود، يمثل

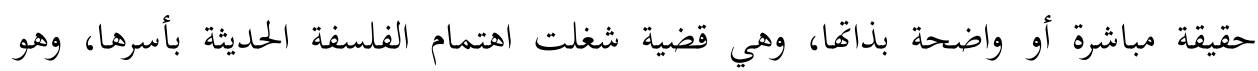
بالفعل يمثل ذلك في نظر واضع هذا المبدأ، أعني ديكارت، وأن الرجل الذي أعلن أن هذه وإه

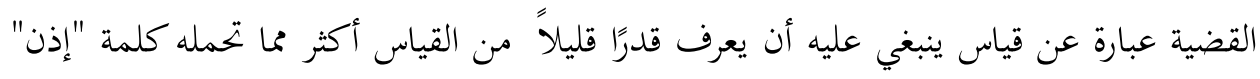
الواردة فيه، فأين يمكن أن نبحث عن الحد الأوسط والحد الأوسط هو أمر جوهري في القياس

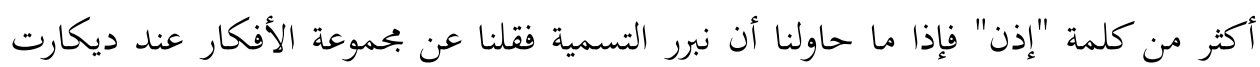
أغها قياس "مباشر" لكان هذا النوع الذي لا لزوم له في القياس هو اسم محض، لمركب غير

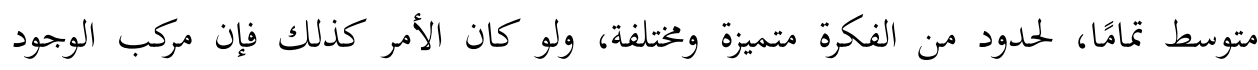
وفكرنا، على نحو ما يتقرر في مبدأ المعرفة المباشرة ليس من حقه أن يحمل اسم القياس أكثر ولا

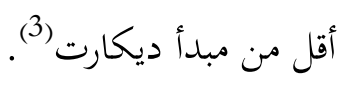

1- مهدي فضل، فلسفة ديكارت ومنهجه، دار الطليعة بيروت، 1983م، ص94.

$$
\text { 2- 20 نفسه ص102. }
$$

3- هيجل، موسوعة العلوم الفلسفية، ترجمة: د.إمام عبد الفتاح، دار الفتوح للطباعة والنشر، بيروت، ط: 1983،

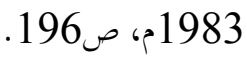


ولكن ديكارت قد رد على مثل هذه الانتقادات ليعلن أن الكوجيتو هو معرفة حدسية

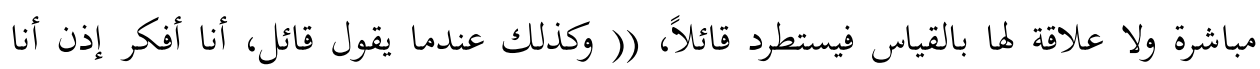

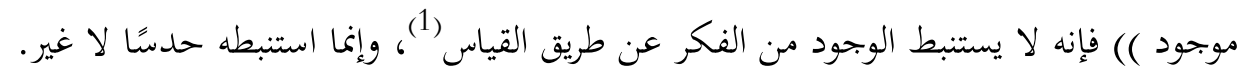

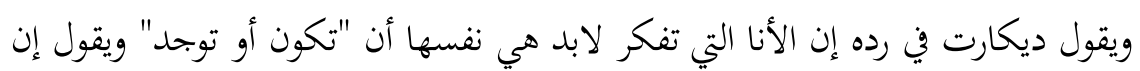

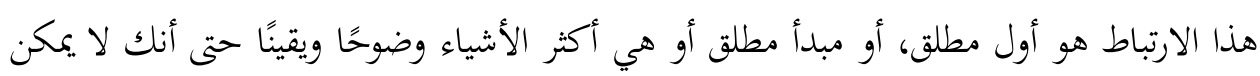

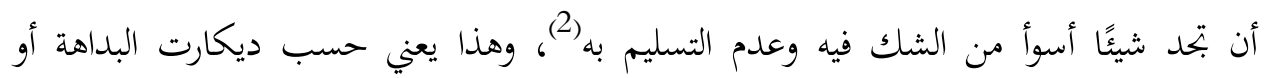
الوضوح. ولكن هيجل يرد على ذلك بقوله إن المعرفة البشرية لا يمكنها أبدًا الاستغناء عنها التوسط، فالمعرفة المباشرة على حد قوله لا تستبعد أبدًا التوسط وأن الاثنين يرتبطان معًا فالمعرفة المباشرة هي بالفعل ناتج المعرفة المتوسطة ونتيجتها (3). ويضرب هيجل أمثلة على ذلك التوسط بقوله، (( لا يقل عن ذلك وضوحًا أن الوجود الفعلي المباشر يرتبط بتوسطه، فالبذرة والآباء عبارة عن وجود فعلي مباشر مبدئي بالنسبة

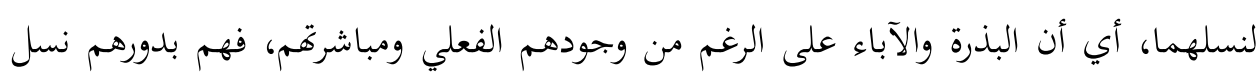

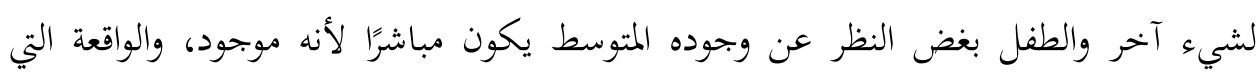
تقول إنني في مدينة برلين، وذلك هو وجودي المباشر الآن، يتوسطها تلك الرحلة الطويلة التي قطعتها حتى وصلت إلى هذه المدينة )(4). ويرى هيجل أن البعض قد يضع تحت ما يُسمى بالمعرفة المباشرة الحدسية ما نطلق عليه الغريزة، والأفكار الفكرية أو المفطورة أو مضمرة في النفس، أوالحس المشترك أو الإدراك العام، أوالعقل الطبيعي، وباختصار أية صورة نعطيها صفة التلقائية، وتعلمنا التجربة العامة أن

$$
\text { 3- 1- ن- نفيجل، مرجع سابق، ص197. نفسه ص199. }
$$


التربية أو التطور مطلوبة لكي يبرز ما هو مطمور داخل الوعي، ولقد كان الأمر كذلك في حالة التذكر عند أفلاطون، وفي طقوس التعبير المسيحية، فرغم أهما مقدسة تتضمن إلزامًا إضافيًّا للتربية المسيحية، وباختصار فإن الدين والأخلاق أيَّا ما كان منهما، إيمان أو معرفة مباشرة، لا يزالان من كل جانب مشروطين بعملية التوسط التي تسمى بالتطور أو التربية أو التدريب(1). وهكذا وجد هيجل أن المعرفة المباشرة لا تستبعد أبدًا التوسط إذا هي تنتقل من الجزئي إلى الكلي ومن الخاص إلى العام، وحتى ما يسمى بالأفكار الفطرية ليست كذلك بل أتت عن طريق التربية والتدريب، ولكن رغم هذه الرؤية التي تنفي المعرفة المباشرة إلا أننا بند من يعلن عن وجود المعرفة المباشرة أو الحدسية داخل المنهج العلمي.

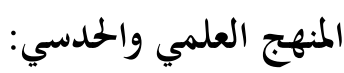

مما لا شك فيه أن خطوات المنهج أو كما يسميها البعض أدوات المنهج العلمي قد أجمع أصحاهما على أها تبدأ بالملاحظة سواء الملاحظة العادية عن طريق الحواس أو الملاحظة عن طريق الآلات، مثل الميكرسكوب والتلسكوب ... إلخ، ثم يليها فرص الفروض، والتحقق من هذه الفروض عن طريق إجراء التجارب واختبار هذه الفروض من أجل الوصول إلى النظرية أو القانون العلمي وهذا هو ما يسمى بالمذهب الاستقرائي في فلسفة العلم. ويستند الاستقرائيون في بناء مذهبهم إلى ثلاثة مبادئ مختلفة تشكل معًا أسس بـ

$$
\text { الاستقراء كمنهج للبحث العلمي وهي، }
$$$$
\text { إن الملاحظة تشكل أساسًا متينًا للمعرفة العلمية. }
$$$$
\text { أننا نتوصل إلى هذه المعرفة العلمية من الملاحظات عن طريق الاستقراء. }
$$

$$
\text { 1- - هيجل، مرجع سابق، ص199. }
$$


وفي الواقع فإن هذه الأركان قد خضعت لمراجعات عديدة من فلاسفة العلم

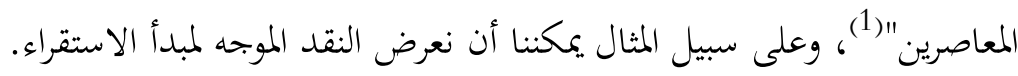

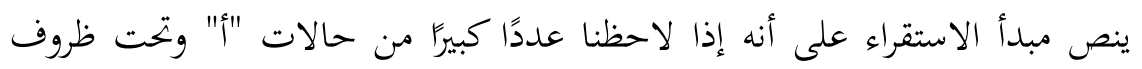
متنوعة، وكانت جميع أفراد "أ" التي تمت ملاحظتها تتمتع بالخاصية "ب" فإِنا لنا نصل على النتيجة

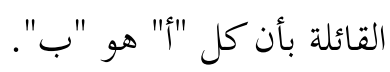
وهذا يعني أنه لكي نصل إلى نتيجة كلية مثل "كل أ هو ب، من ملاحظات جزئية لبعض أفراد "أ" فإنه يلزمنا شرطان جوهريان، الشرط الأول، هو أن نلاحظ عددًا كبيرًا جدًا من إنه

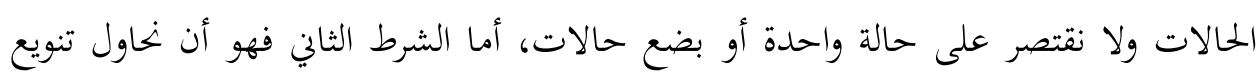

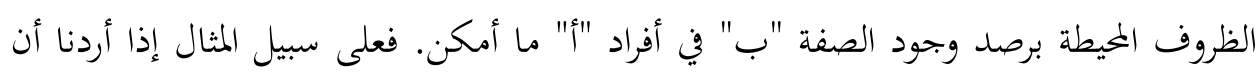
نصل إلى الحكم بأن كل المعادن تتمدد بالحرارة فيجب علينا أن نلاحظ أو بحرب حالات ألات كثيرة

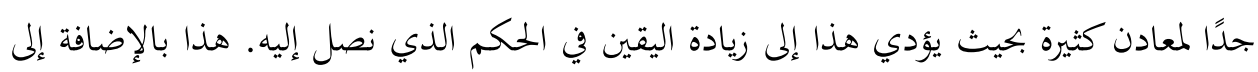

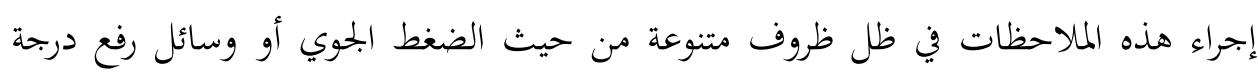
الحرارة وخحلافه (2). ويتسأل الاستقرائيون المعاصرون هل إضافة هذين الشرطين كافٍ لصحة الوثوب من مقدمات الاستقراء إلى نتائجه؟ وكانت الإجابة عند كثير من فلاسفة العلم هي النفي القاطع، حيث سأل بعضهم ما هو العدد الذي يكفي لأن نعتبره كبيرًا من الحالات والملاحظات؟ إن هذا الأمر يختلف فيه الناس تبعًا لأسس شخصية أو نفسية وليست علمية، فإنك إذا أعطيت شخصًا عملية حسابية معقدة بعض الشيء وطلبت منه حلها الحل الأكيد، ستجد أن بعض الناس قد يجري العملية مرة واحدة أو مرتين وعند آخرين وربما أكثر من مرة، وهذا يعكس

1- مختار البسيوني، محاضرات في فلسفة العلوم الطبيعية، وكالة الشرق الأوسط، للطباعة، القاهرة، 2004م، ص 118

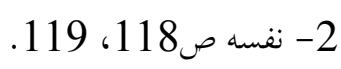


اختلاف الناس في تقديرهم لعدد المرات التي تكفي للحعلنا متأكدين من صحة حل المسألة

$$
\text { الحسابية) (1) }
$$

أما بالنسبة للشرط الثاني وهو تنوع الظروف التي نلاحظ فيها الظاهرة، فإن الاستقراء يقع في مشكلة كبرى، فنحن بند أن قضية تنوع الظروف التي يتحدث عنها لابد أن تكون محكومة باعتبار نظري، وهذا يخرج بنا عن الصورة التقليدية للاستقراء. فالمقياس الذي نقرر به، ما هو مناسب وما هو غير مناسب من حيث تنوع ظروف وشروط الملاحظة العلمية هو مقياس نظري بمعنى أنه من الضروري أن تكون هناك نظرية معينة تقرر في ضوئها أن التنوع في هذا الشرط أو ذاك مناسب لضمان سلامة الانتقال من نتائج الملاحظات إلى التعميم الشامل، وهذا

$$
\text { بالضبط يتناقض مع الصورة المرسومة للاستقراء (2). }
$$

وهكذا تزعزعت أسس الملاحظة كأساس يقين للعلم والمعرفة العلمية واهتزت الأسس

الفلسفية والمنهجية التي يقوم عليها المذهب الاستقرائي في صورته الكلاسيكية، وهنا كان لزامًا على أصحاب المنهج الاستقرائي الذين يتمسكون بأن البحث العلمي يبدأ بالملاحظة أن يدخلوا تعديلاً أساسيًا على منهج البحث العلمي ولذلك سلموا بأن الفروض والنظريات الجديدة يتم ابتكارها أو اكتشافها بطرق عديدة، فبعضها يتم اكتشافه بطريقة مفاجئة فيما يشبه الإلمام وهي المعرفة المباشرة الحمسية- من بحرد حادثة بسيطة وأصدق مثال على هذا هو القصة المتعلقة

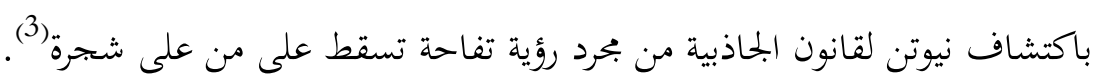
وهكذا بند أن منهج البحث العلمي قد فتح الباب ولو قليلاً لما يسمى بالمعرفة Sir "المباشرة أو الحدسية في بعض الحالات وليس في كل الحالات وهذا هو "سير بيتر مدور

$$
\text { 3- 1 - نغ نفار البسيوني، مرجع سابق، ص120، 121، } 121 .
$$


Peter Medawar الملاحظة لا يمكنه استبعاد حدس العالم، وحدس العالم عنده هو التخيل أو ما يسميه عامل الفن أو المهارة ويزيد بيتر مدور هذا الأمر وضوحًا قائلاً، (( لقد اعتاد الأطباء المرموقون، والأكثر بناحًا وخبرة حسب سجلاقمم أن يشيروا وهم في حدود الحديث عن مهنة الطب إلى أنه رغم الوجود البارز والأساسي لعامل العلم في ممارسة الطب، إلا أن هناك مع ذلك عامل المهارة الفنية ونفاذ البصيرة، ولعل الطب في النهاية ليس إلا تزاوجًا بين الاثنين، عامل العلم وعامل المهارة، إلا أن الخطأ فيما قيل، وكما أراه لا يكمن في حقيقة ذلك التزاوج، وإنما في خلطنا بين العروس والعريس، هو زواج بين

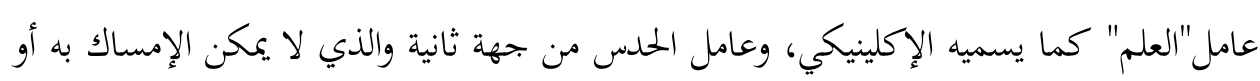
شرحه منطقيًا أو عامل الفن كما تجري تسميته ()) (1). ويحاول "مدور" ضرب الأمثلة من القطاع الطبي وهو ميدان عمله التطبيقي ليثبت أن

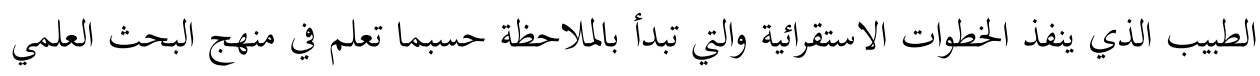

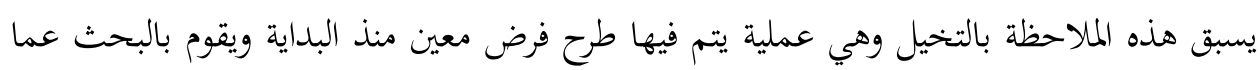

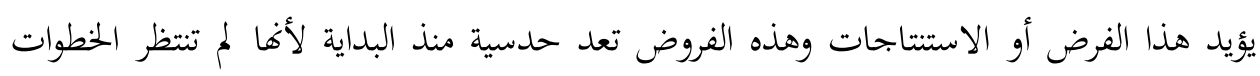
المعهودة للاستقراء العلمي. وهذا هو ما يسميه التبادل السريع بين العملية التخيلية والعملية النقدية، بين الإقدام التخيلي والتريث النقدي ولدى الطبيب تتقدم عملية التشخيص فتتشكل نوع الفرضية التي تبدو رغم

*بيتر مدور، من مواليد الأرجنتين 1916م، من أصل لبناني، هاجرت عائلته إلى إبخلترا في طفولته، بدأت

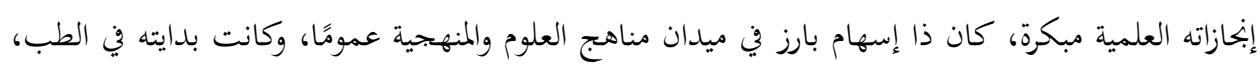

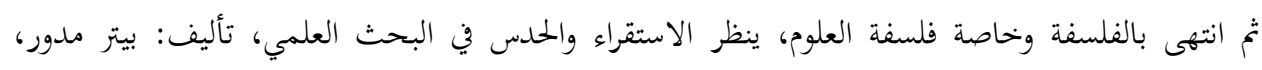

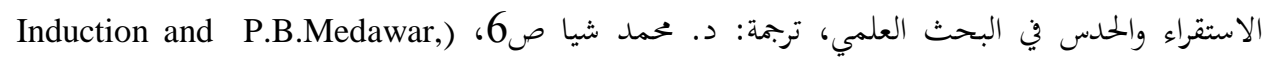
.(Intuition in scientific thought, New Fetter Lane, London 1972 1 - نفسه ص48. n- 
طابعها اللافائي كقاعدة صالحة للمعالجة أو كخطوة ثابتة نحو مزيد من التحقق، والتخيل لا يكون من فراغ، بل يجب أن يكون هناك موضوع للتخيل وهو خطوة أولى تسبق أي بحث أو اكتشاف (1). وهذا التخيل الحدسي الذي يسبق الملاحظة وخطوات الاكتشاف العلمي يسميه مدور

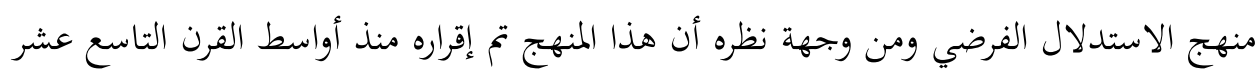
كبديل لاستقراء "جون استيوارت مل" وهذا ما وجد في محاضرات كانط، ولكن المحامي الأكبر

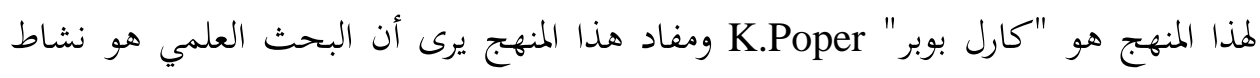
إبداعي يتكون دائمًا من قطبين متعارضين ومتكاملين في آن واحد، أحدهما تخيلي والآخر نقدي، في الجانب التخيلي نحن نشكل رأيًا، وجهة نظر، أو تخمينًا نعتقد أنه يفسر الظواهر

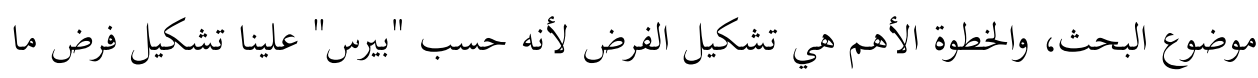
وإلا ضاعت كل معرفة منتظرة أو معرفة جديدة (2).

وهكذا فإن الخطوة الأولى في خط العلم لا تكمن في ما يمكن للحس أن ئنس يقدمه من معطيات (( بل في التصور التخيلي المسبق لما يمكن أن يكون صحيحًا، وبعد هذا التخيل يرى "مدور" أن هذا الأمر نسميه إذا جاز التعبير "الحدس الاستقرائي، وهو أن تصل مباشرة أو فورًا إلى نتيجة منطقية تلي فرضية ما أو تستند إليها، وهي ذي الخطوة الأولى الرائدة في الكشف

$$
\text { (العلمي () (3) }
$$

وهكذا قدم لنا "مدور" في ميدان البحث العلمي ما يسميه الحدس الاستدلالي، وهو

التقاط النتائج في برهة أو لحظة من الزمن، أي أن ندرك على نهو فوري النتائج المتوقعة من

$$
\text { فرضية أو رأي مال). }
$$

$$
\begin{aligned}
& \text { 1- بيتر مدور، مرجع سابق ص49. } \\
& \text { 2- نفسه ص49، } 40 . \\
& \text { 3- نفسه ص54، } 57 \text { نفسه ص } 57 . \\
& \text { 4- نفسه ص58. نغسه ص.5 }
\end{aligned}
$$


وأن "مدور" قد أعطى للتخيل أو المخيلة دورًا أساسيًّا في المعرفة العلمية فلقد سبقه "بشلار" الفيلسوف الفرنسي الذي توفي عام 1963م بفرنسا، فقد أكد على دور المخيلة في المعرفة العلمية وخاصة في تحديده للمعرفة العلمية بأها معرفة علاقات(1) بمعنى أها معرفة عقلية قبل أن تكون بتريبية حسية. فالمخيلة ليست بالضرورة ملكة خلق الصور فحسب؛ بل -أيضًا- القدرة على إدراك علاقات جديدة، لهذا السبب يعتبر بشلار أن عقلانية العلم ليست فلسفة مبادئ قارة بل هي تقتضي التحوير المستمر لمبادئ العلم، فحركة التحوير التي توجه المعرفة حركة جدلية وهي تخص المعرفة من حيث هي بناء نظري وصيرورة تاريخية. إن جدلية المعرفة تعني ارتباط الواحد بالآخر. فالصور التي تبدعها المخيلة ضرورية لبناء المعرفة(2). ويفصل باشلار في دراسة العلم بين مستويين اثنين، مستوى الاكتشاف ومستوى المراقبة، ففي مستوى الاكتشاف يصعب التمييز بين ما هو علمي وما هو غير علمي وفي هذا المستوى لا يمكن أن نستند إلى الحقيقة بين المعرفة العلمية والمعرفة التي تفتقر إلى هذه الصفة، فالنقد لا يكون له أي تأثير إذا لم تقدم المخيلة مادة أولى للمعرفة. فدور النقد ثانوي بالقياس للاكتشاف أو أن المخيلة هي التي تكتشف أما النقد فيكتفي بالمراقبة(3). وهكذا فإن المخيلة وهي معرفة حدسية مباشرة تسبق البرهان وتسبق الاستدلال، وهي معرفة يقدمها الاستقرائيون المعاصرون على مبدإ الاستقراء التقليدي. وإذا انتقلنا إلى ميدان آخر بعيدًا عن منهج البحث العلمي لوجدنا أن هذه المعرفة الحدسية انتقلت إلى ميدان الأخلاق والقيم الأخلاقية.

1- فاستونباشلار، حدس اللحظة، ترجمة: رضا عزوز، دار الشؤون الثقافية العامة، العراق، "د. ت"، ص98. 2- نفسه. -3 - نفسه. 


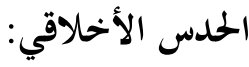

عرفنا فيما قبل أن الحلدس هو فهم مباشر لموضوع بدون توسط أي عملية استدلالية،

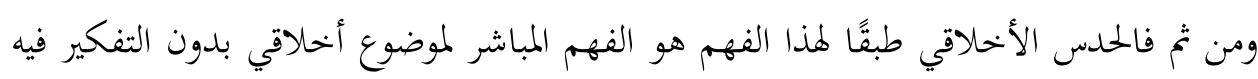

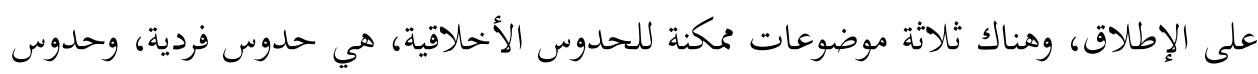
عامة، وحدوس فلسفية (1).

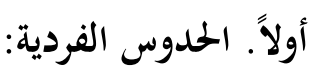

وتعني أن بإمكاننا أن نعرف مباشرة أن فعلاً بعينه مثل اغتيال بروتس لقيصر كان فعلاً

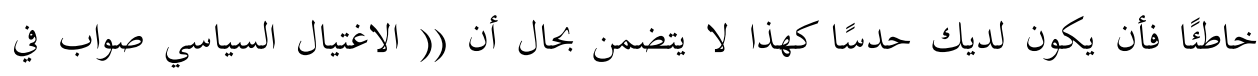
الحالات الأخرى، وقد وصف "سد جويك" Sidgwick النظرية التي ترى أن السبيل الوحيد

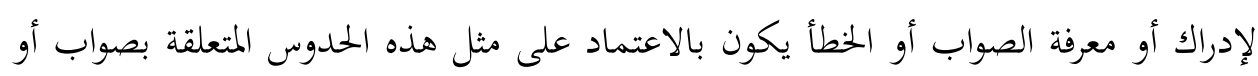

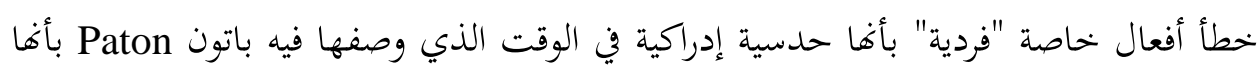

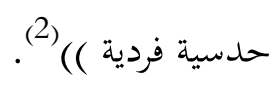

فعندما يرى المرء مباشرة أن فعلاً بعينه صواب بالنسبة له في هذه اللحظة الراهنة، ولا يكون لديه فرصة لإجراء مزيد من التأمل ودراسة الحالة، فإن أغلب فلاسفة الأخلاق سيوافق على أن واجب المرء هنا أن يعمل بحسب ما يقضي حدسه، وتستطيع أن تقول أن هناك اعتبارين يؤيدان هذه الوجهة من النظر، آنساء أن في كل مناشط الفاعلية الإنسانية يقوم المرء بتخليق عادات للفعل وتمنحه هذه

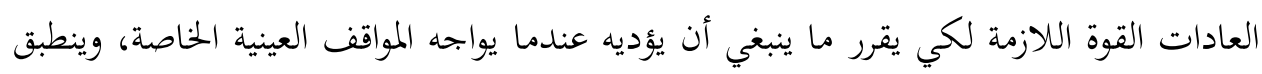

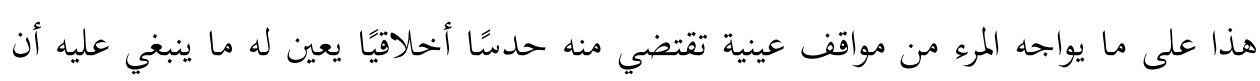
يؤديه.

1- وليام ليلي، المدخل إلى علم الأخلاق، ترجمة د. علي عبد المعطي، دار المعرفة الجامعية، الإسكندرية،

$$
\text { 1991 1991 ص219. }
$$

2- د. محمد مدين، الحدس الخلقي، دار الثقافة للنشر والتوزيع، القاهرة، 1990م، ص17. 
أن الموقف الخلقي قد يكون، وإلى حد ما موقغًا متميزًا، فنحن قد نستجيب للمواقف

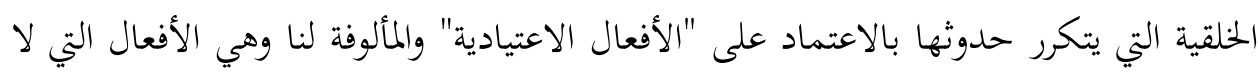
تحتاج منا جهادًا تأمليًا أو قد تستجيب لهذه المواقف بتطبيق قاعدة ما من القواعد الأخلاقية المستقرة، ولكن الموقف الجديد هو الذي يوقظ فينا "الضمير" أو "الوعي" ويحثه لاتخاذ قرار

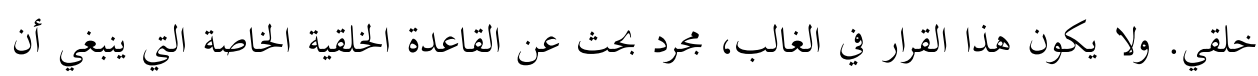
تطبق في هذا الموقف الجحديد(1).

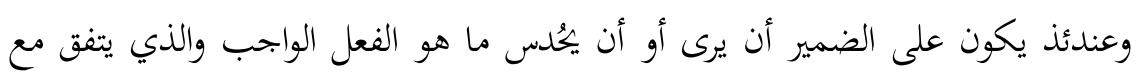
الظروف الجديدة والتي ربما تكون فريدة ومتميزة وهذا الكشف لما هو واجب في الحئل الحالة الخاصة والعينية

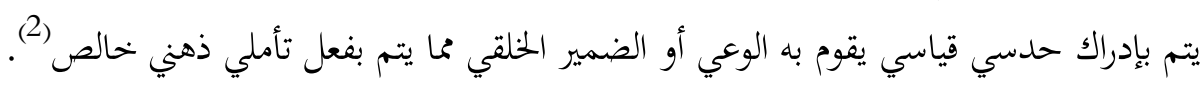

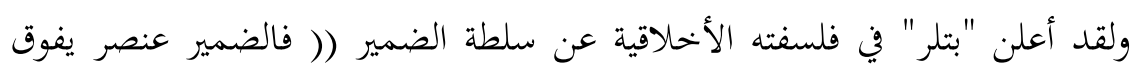
طبيعيّا مبدأ حب الذات ومبدأ فعل الخير وقراراته غهائية ومباشرة وحدسية، وإن كان "بتلر" اعتبر فئه

$$
\text { الضمير حدسيًّا من جهة وعقليَّا من جهة أخرى ()) (3). }
$$

والموقف الأخلاقي الفردي الذي يستخدم فيه المرء حدسه الفردي قد يكون موقغًا

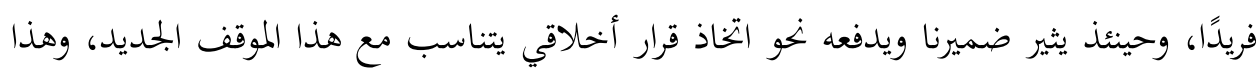

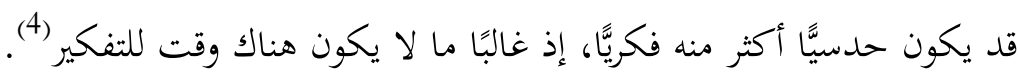

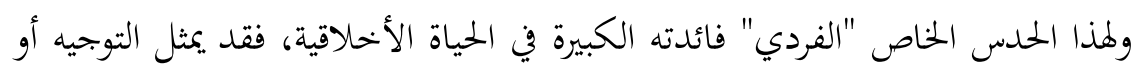
الإرشاد الأوحد لشخص ما في موقف يتطلب اتخاذ إجراء فوري، وهو الذي يواجه المواقف

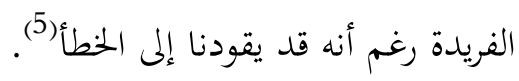

$$
\begin{aligned}
& \text { 1- د. محمد مدين، ص17، } 18 . \\
& \text { 2- نفسه. } \\
& \text { 3- وليام ليلى، مرجع سابق ص224. } \\
& \text { 4- نفسه ص225. } \\
& \text { 5- وليام ليلى، مرجع سابق ص225. }
\end{aligned}
$$




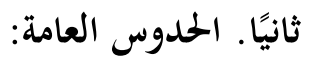

يمكننا أن نعرف وعلى نهو مباشر، وبدون عمليات استدلالية أن "فئة" أو "نوع" معين من الأفعال هي أفعال صائبة أو خاطئة كما في حكمنا (( أن قول الصدق على

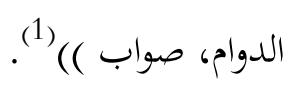

ولكن "سدجويك" أشار إلى أن مثل تلك القواعد الحدسية لا تكون صادقة في كل الحالات فهناك قواعد يمكن الشك فيها، وهناك قواعد أخرى لا تكون صادقة بالمرة، فالقتل خطأ، لكن القتل دفاعًا عن النفس ليس كذلك، ويبالغ "سد جويك" قائلاًٌ إن الحدوس العامة لا تكون حدوسًا على الإطلاق؛ بل هي من وجهة نظره بحرد تعميمات اشتققناها من خبرتنا بأنماط السلوك التي تتأدى بنا إلى سعادتنا العامة في المختمع (2). ولقد أكدت المدرسة الحدسية الاسكتلندية التي تزعمها "توماس ريد" على أن المبادئ الخلقية قبلية سابقة على التجربة وأها موضع تسليم من الناس في كل زمان ومكان، تلك هي مبادئ الحس المشترك Common Sense وبهذا أدركوا وجود إدراك حدسي يمكن الإنسان من التمييز بين الصواب والخطأ أو الخير والشر، فتنتفي بهذا حاجة الناس إلى حاكم مطلق يقوم

$$
\text { بتحديد الخير والتمييز بينه وبين الشر(3). }
$$

والمقصود بالحدوس الفلسفية هو الرأي القائل بأن المبادئ الأخلاقية التي نصل إليها بواسطة الحدس ليست نظرية أخلاقية ؛ بل نظرية فلسفية تستهدف الوصول إلى الحقائق الكلية، وأن ما نعرفه بواسطة تلك الحدوس ليس صواب أو خطأ الأفعال الخاصة أو طائفة معينة من

$$
\text { 3- } 1 \text { - ت- وليام ليلى، مريق مدين، مرجع سابق صابق ص226. }
$$


تلك الأفعال، لكن ما نعرفه هو بعض القواعد أو المبادئ التي تساعدنا بطريق مباشر أو غير مباشر على اكتشاف ما إذا كان الفعل صائبًا أو خاطئًا(1). لقد وصف "سدجويك" هذه الحدوس بأها فلسفية، وهو يعني بذلك أها نظرية في

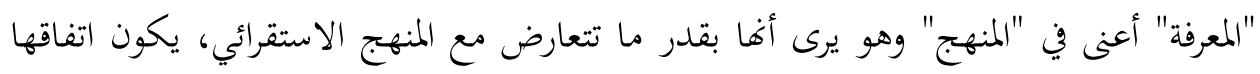
مع المنهج الاستنباطي (2). فمن المعروف أن هناك نظريتين في المعرفة، النظرية الاستقرائية التي نبدأ بها بملاحظة الوقائع الجزئية وإقامة تعميمات ابتداء منها على هيئة قضايا كلية، والنظرية الاستنباطية التي نبدأ

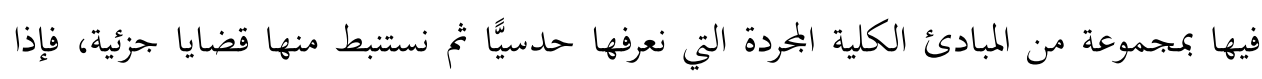

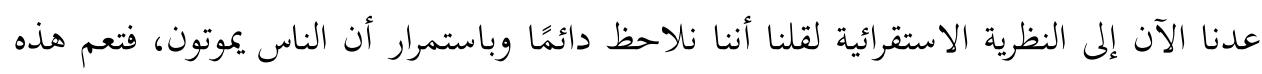
الملاحظة ونصل إلى القضية الكلية "كل إنسان فان" وبنفس الطريقة نصل إلى القضايا الوصفية مثل "أن كل الأجسام الأثقل من الهواء تتجه إلى السقوط بحاه الأرض" بيد أن الملاحظة وحدها

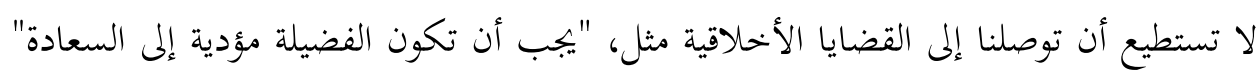

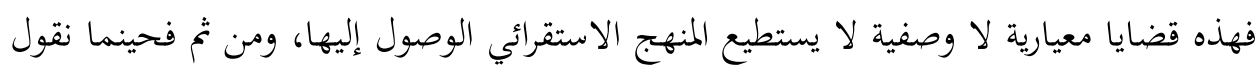

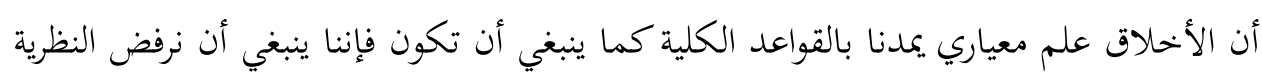
الاستقرائية التجريبية(3).

وأما المنهج الاستنباطي فيؤكد في أكثر صوره شيوعًا على أن كل معرفتنا تعتمد على ألى المبادئ المحردة التي نعرفها بالحدس المباشر، ومن ثم إذا أردنا أن تظل الأخلاق علمًا معياريًا

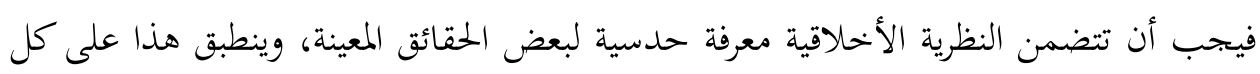

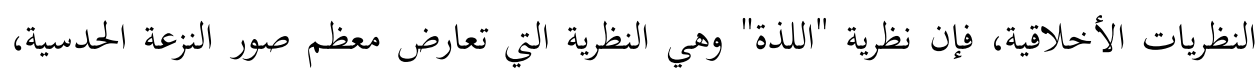

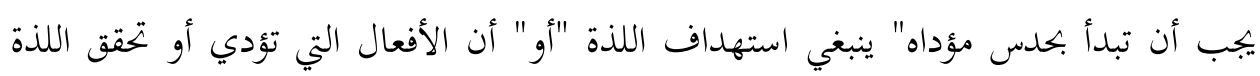

$$
\begin{aligned}
& \text { 1- وليام ليلى، مرجع سابق ص228. }
\end{aligned}
$$

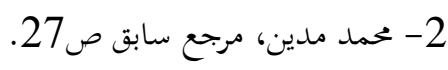

$$
\begin{aligned}
& \text { 3- وليام ليلى، المرجع السابق صد228. مرجع لسابق صدان. }
\end{aligned}
$$


هي وحدها الأفعال الصواب (( فليس هناك ثمة طريق آخر سوى الحدس لاكتشاف المبدأ الأساسي لنظرية اللذة أو أي نظرية أخرى؛ وذلك لأن المبدأ هنا هو ما نحن بحاجة إلى ألى أن نرى النى بالإدراك المباشر أنه واضح بذاته )(1). وهكذا فإن قواعد الأخلاق في المذهب الحدسي واضحة بذاتها لأها لا تحتمل برهانًا ولا تقبل تبريرًا، والمعروف أن الناس على اتفاق في أن السعادة والحكمة وغيرها من الكمالات فضائل،

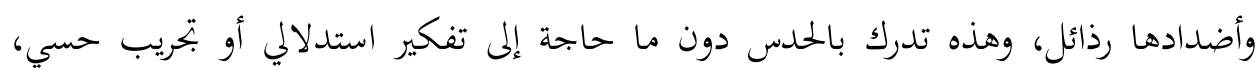

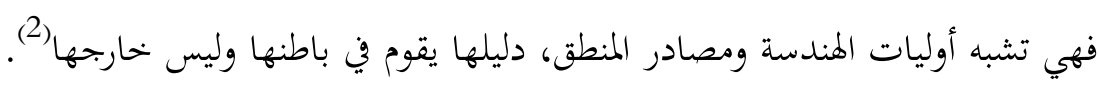

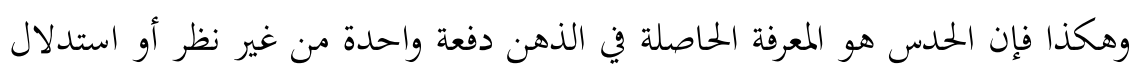
عقلي، وهذا المعنى الذي أخذ به "شوبنهاور" لا يصدق على تمثل الأشياء فحسب؛ بل يصدق أيضًا على تمثل علاقاتما كمثل خواص الأعداد والأشكال الهندسية من جهة ما هي مدركة إدراكًا مباشرًا، وأكمل صور الحدس عنده الحدس الجمالي، الذي ينسى فيه الإنسان نفسه في لحظة

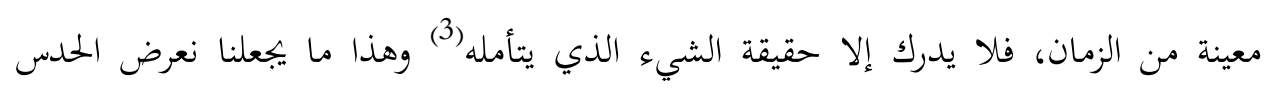

$$
\text { الفني، خاصة عند "كروتشه". }
$$

لقد أعلن الفيلسوف "كروتشه" باعتباره أحد أقطاب الفلسفة الحدسية في ثنايا تعريفه للفن بأنه حدس فأجمل ذلك بقوله، (( وحسبنا أن نقول أن الفن حدس حتى نعرف الفن أكمل تعريف" وكذلك أن كل خلق فني حقيقي هو حدس محض، ثم يعلق في نص آخر بأن الفن لا يمكن أن يكون أبدًا إلا حدسًا محضًا () (4).

$$
\begin{aligned}
& \text { 1- محمد مدين، مرجع سابق ص28، } 29 .
\end{aligned}
$$

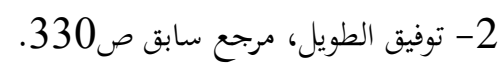

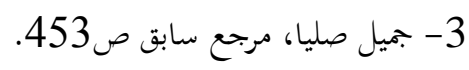

$$
\begin{aligned}
& \text { 4- عبد الكريم هلال، أسس النقد الجمالي في تاريخ الفلسفة، 2003 صات صمان ص131. }
\end{aligned}
$$


ولا يمكن تفسير الفن ولا بحريده من غيره من المفاهيم وبالتالي حصره في نطاقه الروحي والمثالي الضيق، فالفن هو حدس محض، ويمكن الإشارة إليه كشيء ميز وحسب. ويسعى كروتشه إلى إبعاد مفهوم المادة عن الفن، فإن حدسية الفن تنكر أولاً أن يكون الفن إنهاء واقعة مادية، أن يكون، مثلاً، ألوانًا أو نسبة بين الألوان، أن يكون أشكالاً جسمية، أن يكون أصواتًا أو نسبًا بين أصوات، أن يكون ظاهرات حرارية أو كهربائية، أي أن يكون على الجملة شيئًا مما يشار إليه بقولهم "مادي" (1).

لكن من ناحية أخرى لا يجب رفض هذه الأشياء رفضًا قاطعًا حيث يستخدمها الحدس الفني كوسائل له يخرج من خلالما، وكوفا وسائل أو أدوات ظاهرة للعين المحردة لا يجعلها تمثل المفهوم الحقيقي لإبداعات الفنية الجمالية، فالمواد المختلفة التي يصنع منها الحدس شكله الخاص تعد وسيطًا بين الفن كروح أو كفكر وبين الحواس التي لا تدرك إلا شيئًا ماديًا ومحسوسًا (2.)

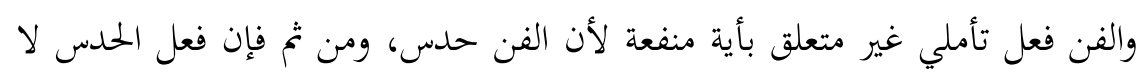
يضع في اعتباره الانتفاع المادي كأي فعل نفعي آخر ومن تم من لا علاقة له باللذة والألم من حيث هما لذة وألم (( أي أغما ليسا أساسًا للحكم أو معيارًا فالحدس لا يتضمن مثل هذا

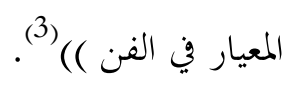
وهذذا يكون الحدس الفني هو الجانب الفني للروح بحيث يكون كل حدس فنًّا، والعلاقة بين الحدس والفن لا تعدو كوغا دائرة مفرغة لأن أحدهما يفسر الآخر (4).

$$
\begin{aligned}
& \text { 1- عبد الكريم هلال، ص132. } \\
& \text { 2- نفسه. } \\
& \text { 3- نفسه ص133. نفسه. } \\
& \text { 4- نفسه } 135 .
\end{aligned}
$$




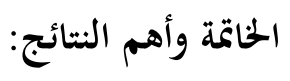

تم التوصل إلى معرفة مفهوم الحدس والشرح للحدوس وأنواعها وتقسيم المواقف المختلفة

بين الفلاسفة الذين أثبتوا المنهج الحدسي في المعرفة وفلاسفة في بحال الأخلاق والفن.

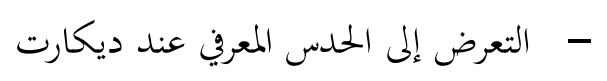

- - المعرفة المباشرة عن هيجل لا تستبعد التوسط فهي تنتقل من الجزئي الم الكلي

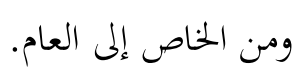

- منهج البحث العلمي وتعامله في بعض الحالات مع المعرفة المباشرة والحدسية. - - المخيلة هي معرفة حدسية مباشرة تسبق البرهان وتسبق الاستدلال، هي معرفة يقدمها الاستقرائيون المعاصرون على مبدأ الاستقراء التقليدي. - الحدس هو المعرفة الحاصلة في الذهن دفعة واحدة من غير نظر أو استدلال عقلي. - الحدس الفني هو الجانب الفني للروح بحيث يكون كل حدس فنًا المنهج العلمي

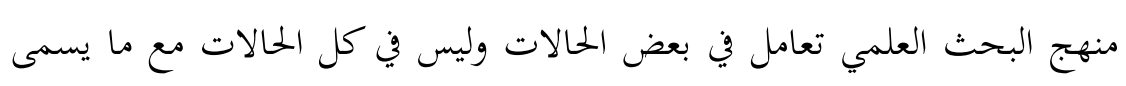
بالمعرفة المباشرة أو الحدسية. 


\section{المصادر والمراجع}

1- بيتر مرور، الاستقراء والحدس في ابحث العلمي، ترجمة: محمد شيا، لندن 1972م.

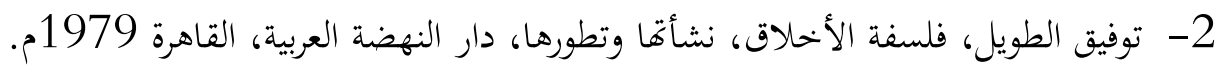
3- جميل صيليبيا، المعجم الفلسفي، دار الكتب اللبناني، ج: 2، بيروت 1971م. 4- ديكارت، التأملات في الفلسفة الأولى، ترجمة: عثمان أمين، مكتبة الأبحلو المصرية، القاهرة. 5- رواية عبد المنعم عباس، ديكارت والفلسفة العقلية، دار المعرفة الجامعية، الإسكندرية 1990م. (200 - n

6- علي عبد المعطي، دراسات في الفلسفة العامة، دار الثقافة للنشر والتوزيع، القاهرة. 7- عبد الكريم هلال، أسس النقد الجمالي في تاريخ الفلسفة، منشورات جامعة قاريونس، بنغازي، ط: 1: 1 1003، 2003.

8- فاستونباشلار، حدس اللحظة، ترجمة، رضا عزوز، دار الشؤون الثقافية العامة، العراق.

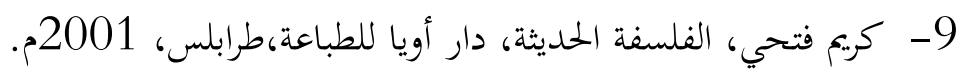
10- مهددي فضل ، فلسفة ديكارت ومنهجه، دار الطليعة، بيروت، 1983 1983م.

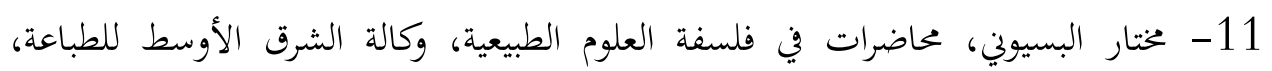
القاهرة، 2004م.

12- محمد مدين، الحدس الخلقي، دار الثقافة للنشر والتوزيع، القاهرة 1990م. 13- مراد وهبة وآخرون، المعجم الفلسفي، مكتبة يوليو، القاهرة.

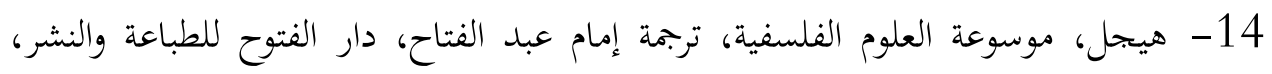

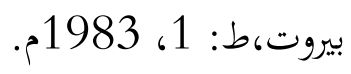
15- وليام ليلي، المدخل إلى علم الأخلاق، ترجمة: د. علي عبد المعطي، دار المعرفة الجامعية، الماف،

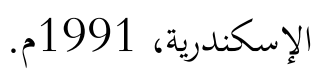
16- يوسف كرم، تاريخ الفلسفة الحديثة، دار المعارف بمصر، القاهرة،ط: 4، 1966م. 